\title{
Self-sustained activity in a small-world network of excitable neurons
}

\author{
Alex Roxin ${ }^{+}$, Hermann Riecke ${ }^{+}$and Sara A. Solla* \\ ${ }^{+}$Engineering Science and Applied Mathematics, * Department of Physiology and Department of Physics and Astronomy, \\ Northwestern University, Evanston, IL 60208, USA
}

(Dated: September 10, 2018)

\begin{abstract}
We study the dynamics of excitable integrate-and-fire neurons in a small-world network. At low densities $p$ of directed random connections, a localized transient stimulus results in either selfsustained persistent activity or in a brief transient followed by failure. Averages over the quenched ensemble reveal that the probability of failure changes from 0 to 1 over a narrow range in $p$; this failure transition can be described analytically through an extension of an existing mean-field result. Exceedingly long transients emerge at higher densities $p$; their activity patterns are disordered, in contrast to the mostly periodic persistent patterns observed at low $p$. The times at which such patterns die out are consistent with a stretched-exponential distribution, which depends sensitively on the propagation velocity of the excitation.

PACS numbers: 05.45.-a,87.18.Sn,87.10.+e
\end{abstract}

Recent research in complex networks has provided increasing evidence for their relevance to a variety of physical, biological, and social phenomena 1, 2, 3]. Two distinct types of topology have been particularly useful in providing insights into the implications of complex connectivity: scale-free networks [3], characterized by the existence of a small number of hubs with high coordination number, and small-world networks [1], characterized by the presence of shortcuts that link two randomly chosen sites regardless of the distance between them.

So far, most work on complex networks has focused on their topological and geometrical properties; less attention has been given to the properties of dynamical systems defined on such networks. The interplay between the intrinsic dynamics of the constituent elements and their complex pattern of connectivity strongly affects the collective dynamics of the resulting system. For instance, the addition of shortcuts induces a finite-temperature phase transition even in the one-dimensional Ising model 4] and the introduction of unidirectional shortcuts can change the second-order phase transition in the twodimensional Ising model into a first-order one [5]. In a system of coupled oscillatory elements, the introduction of shortcuts enhances synchronization [6], while the introduction of hubs eliminates the threshold for epidemic propagation [7].

The coexistence of shortcuts and regular local connections characteristic of small-world networks (SWNs) mimics a salient feature of the circuitry in the cortex 8, 9, 10, 11, 12, 13], where experimental observations of excitatory traveling waves [11] provide evidence of some degree of local connectivity, while it is also recognized that long-range excitatory connections are present [10, 12]. Our goal is to explore the influence of this complex connectivity on the dynamics of neuronal circuits; to this purpose, we choose a minimal model. The underlying network is modeled as a SWN with unidirectional shortcuts that reflect the nonreciprocal character of synaptic connections, and the excitable neurons are modeled as leaky integrate-and-fire units. We find that even this simple model exhibits a rich repertoire of distinct dynamical behaviors as a function of the density $p$ of added shortcuts: a low $p$ regime characterized by persistent periodic activity that is bistable with the quiescent state, a transition to failure with increasing $p$, followed by a reemergence of long-lasting disordered activity. We note that a SWN with unidirectional shortcuts has been considered in a different regime by Lago-Fernández et al $[14,15]$ to address the possibility of rapid synchronization among conductance-based neurons of the HodgkinHuxley type.

The model considered here consists of a onedimensional array of $N$ integrate-and-fire neurons (IFNs) in which a SWN topology is created through the addition of a density $p$ of unidirectional long-range couplings. The membrane potential of the IFNs is determined by

$$
\tau_{m} \frac{d V_{i}}{d t}=-V_{i}+I_{e x t}+g_{s y n} \sum_{j, m} w_{i j} \delta\left(t-t_{j}^{(m)}-\tau_{D}\right)
$$

A neuron fires whenever its voltage exceeds 1; the voltage is then reset to 0 . The external current is chosen to satisfy $I_{\text {ext }}<1$; in this regime the IFNs are not oscillatory, but excitable. The last term models input currents due to presynaptic firing as a delayed impulse: if $w_{i j}$ is non-zero, then neuron $i$ receives a pulse input of amplitude $g_{s y n}$ with a delay $\tau_{D}$ after neuron $j$ has fired its $m$ th spike at time $t_{j}^{(m)}$. The synaptic conductance is chosen to satisfy $I_{\text {ext }}+g_{\text {syn }}>1$, so that a single input suffices to sustain firing activity. The local connections are modeled here as nearest-neighbor couplings $\left(w_{i, i \pm 1}=1\right)$ that define an underlying regular lattice. The long-range connections result from randomly adding rather than rerouting [1] a fixed fraction $p N$ of unidirectional couplings $w_{i j}=1$ to generate a SWN topology.

At $p=0$, any excitation sufficient to cause a neuron to fire will generate two pulses that propagate through the regular lattice in opposite directions with velocity $v=1 / \tau_{D}$, and either exit the system or annihilate each other, depending on boundary conditions. No persistent 

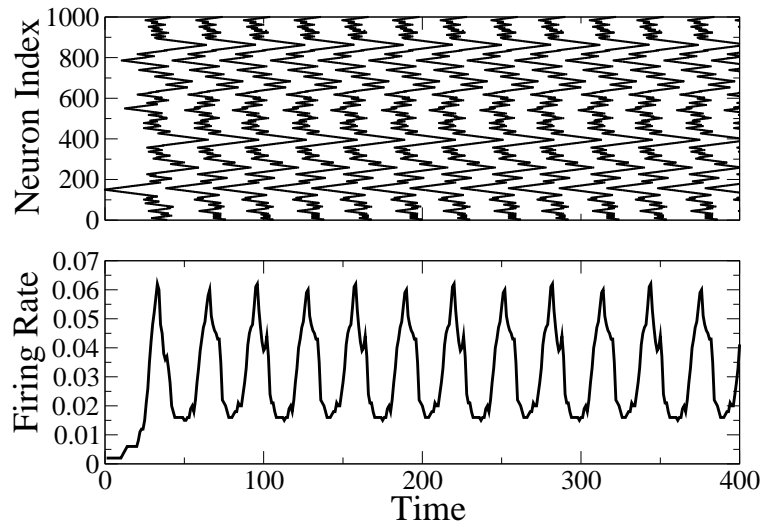

FIG. 1: Raster plot (top) and instantaneous firing rate (bottom) for a system with $N=1000, I_{e x t}=0.85, g_{s y n}=0.2$, $\tau_{m}=10, \tau_{D}=1, p=0.1$. Same parameter values are used in subsequent figures unless noted otherwise.

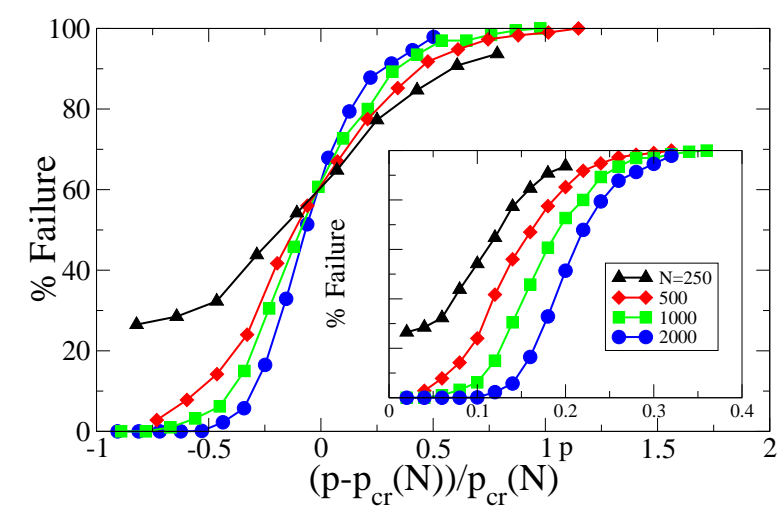

FIG. 2: Inset: Failure rates for $\tau_{D}=1.0$ and different system sizes $N$. Main: Failure rates normalized by $p_{c r}(N)$ (cf. (34)).

activity results in either case. However, self-sustained activity may arise for nonzero $p$, as shown in fig 1 Persistence relies on the reinjection of activity via a shortcut into a previously active domain that has by then recovered; this reinjection can occur only if the shortcuts are unidirectional. For a fixed value of $p$, any particular network realization has a different connectivity graph that may or may not sustain persistent activity. We average over typically 2000 realizations to calculate the probability of persistent activity; the complementary probability of failure to sustain activity is shown in fig (inset) as a function of the density $p$ of random connections for four different system sizes. In this regime, the probability of failure is an increasing function of $p$ that crosses over from 0 to 1 with increasing steepness as the size $N$ of the system increases.

Failure to sustain activity is a simple consequence of the intrinsic dynamics of the neurons. Pulses travel outwards from an initial activity seed, and spawn new pulses at a rate that increases with $p$. A currently inactive neuron can fire again only if enough time has elapsed from its preceding firing to allow for a recovery to $V \geq 1-g_{s y n}$.

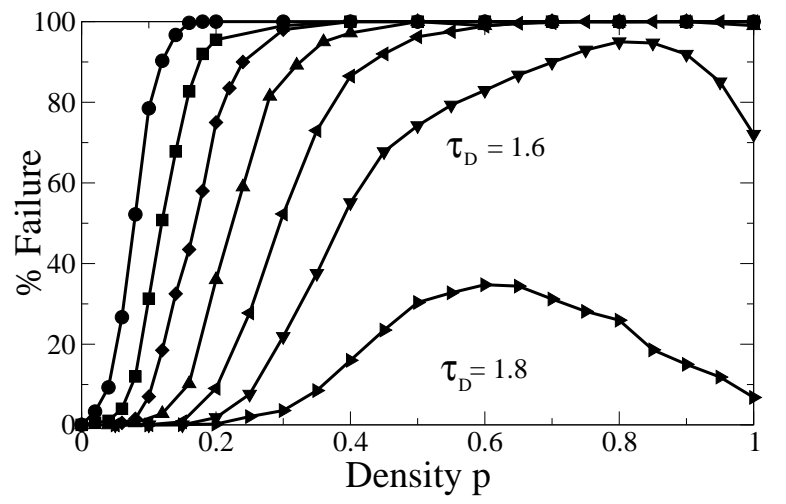

FIG. 3: Failure rates after $t_{\max }=2000$ for $\tau_{D}=0.6,0.8,1.0$, $1.2,1.4,1.6,1.8$ (left to right).

A single input will be able to elicit a spike only if the elapsed time exceeds $T_{R}^{(1)}$, with

$$
T_{R}^{(n)}=\tau_{m} \ln \left(\frac{I_{e x t}}{I_{e x t}+n g_{s y n}-1}\right) .
$$

If activity recurs to a given site too rapidly, the neuron will fail to produce a spike, and the pulse of activity will die out. A critical density $p_{c r}$ for the transition from self-sustained activity to failure can be estimated from

$$
T_{A}\left(p_{c r}\right)=T_{R}^{(1)},
$$

where $T_{A}(p)$ is the time needed for the activity to spread across the whole network. At a fixed velocity for pulse propagation, this time corresponds to the largest distance across the network. This distance has been calculated for bidirectional shortcuts using a mean-field approach [16]; when extended to the case of unidirectional shortcuts it results in

$$
\sqrt{\left(1+\frac{4}{p N}\right)} \tanh \left[\sqrt{\left(1+\frac{4}{p N}\right)} \frac{p T_{A}(p)}{2 \tau_{D}}\right]=1 .
$$

An implicit expression for $p_{c r}$ and its dependence on the system size $N$ and the propagation velocity $v$ follows from combining equations (31) and (4); for large $N, p_{c r}(N) \propto \ln N$. Failure rate curves as a function of $\left(p-p_{c r}(N)\right) / p_{c r}(N)$, shown in fig 2 cross at the theoretically predicted value. This observation, together with the increased steepness of these curves with increasing $N$, indicates that a well-defined transition to failure occurs in the thermodynamic limit.

This well-defined transition to failure occurs only for sufficiently fast waves, i.e. for short delay $\tau_{D}$. For larger $\tau_{D}$ the dynamics of the system become quite more complex, and the fraction of realizations that fail before a fixed time $\left(t_{\max }=2000\right.$ in fig 3 ) becomes a nonmonotonic function of $p$. While at low $p$ the firing patterns are highly regular (cf. fig 1 ) and all failures occur within one or two cycles of the initial activity, for higher $p$ the patterns are more disordered (cf. fig凷) and the activity 

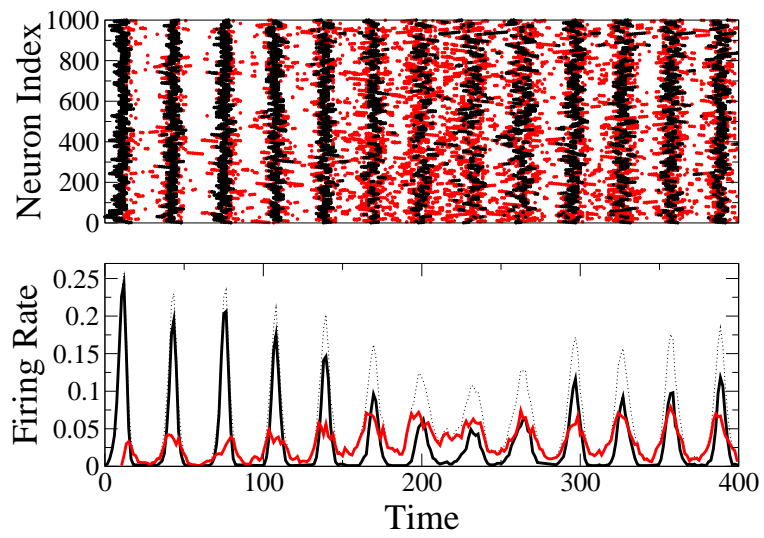

FIG. 4: Raster plot (top) and instantaneous firing rate (bottom) of neurons with $I S I>T_{R}^{(1)}$ (black) and $I S I<T_{R}^{(1)}$ (red) for $\tau_{D}=1.5$ and $p=1.0$. Dotted line is the total firing rate.

can persist for a very long time before failure. Consequently, the distribution of failure times, shown in fig 5 exhibits an increasingly long tail for longer delay times.

To understand the persistence of activity beyond $p_{c r}$, it is important to recognize that the result for the critical density $p_{c r}(N)$ hinges on the assumption that each neuron receives but a single excitatory input during each cycle of network activity. Its recovery time is therefore given by $T_{R}^{(1)}$, which sets a lower bound for the interspike interval (ISI). While this assumption is well satisfied for small $p$, it does not hold for $p=O(1)$. In fact, the likelihood that a neuron has $n$ incoming shortcuts follows a multinomial distribution such that the fraction of neurons receiving two incoming connections grows from about $0.05 \%$ at $p=0.1$ to about $30 \%$ at $p=1$. Neurons that receive $n$ inputs can have a recovery time as short as $T_{R}^{(n)}$. Fig 4 reveals that such neurons, with ISIs lower than $T_{R}^{(1)}$, play a crucial role in maintaining network activity in the regime $p \approx 1$. While neurons with ISIs greater than $T_{R}^{(1)}$ (marked black in fig 4 ) can go through silent epochs with near-zero activity, neurons with shorter ISIs (marked red in fig (4) may fire two or three times during a network cycle and carry over the network activity across these silent epochs.

The persistence of activity beyond $p_{c r}$ depends sensitively on $\tau_{D}$; this reflects the fact that persistence at higher densities $p$ is due to chains or trees of neurons that bridge the silent epochs due to their multiple inputs. Since activity propagates with a fixed speed, a chain of multiple-input neurons of given length can bridge a time interval proportional to $\tau_{D}$. Thus, as $\tau_{D}$ increases, ever shorter chains can contribute to bridging a silent epoch of given duration; the likelihood for failure will decrease accordingly. This picture is of course overly simplistic: whether a topological chain can be utilized as a dynamical bridge over a given silent epoch depends on the amount and timing of the inputs it receives, which in turn depend on the recent history of the entire network. Simu-

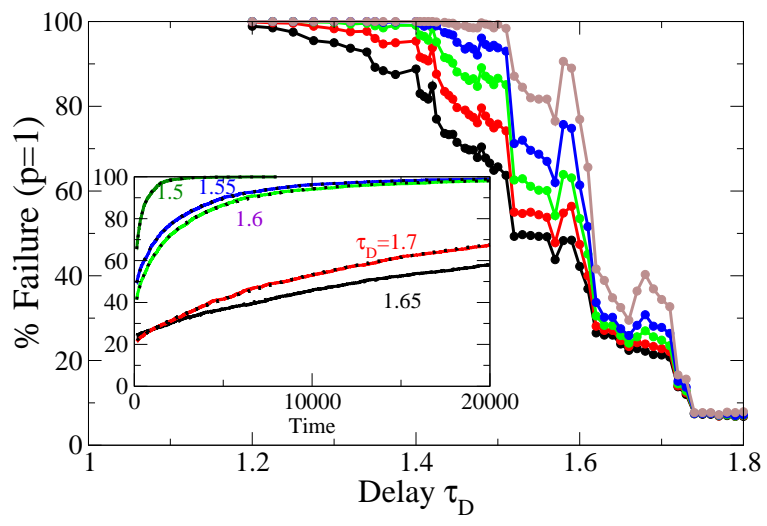

FIG. 5: Failure-time distribution. Inset: Cumulative distribution of failure times at $p=1$ for $1.5 \leq \tau_{D} \leq 1.7$. Main: Failure rates at 5, 10, 20, 40 and 100 multiples of $T_{R}^{(1)}=28.3$ (black to brown). Symbols are averages over 2000 realizations.

lations reveal that the identity of the neurons that form the 'bridging' dynamical chains varies from cycle to cycle in an irregular way. This implies that the effective utilization of a dynamical chain over one cycle does not guarantee its availability on the next cycle. Therefore, even systems that persist for long times may still have a finite probability of failing.

The cumulative failure distribution function $F(t)$, shown in fig 1 for various values of $\tau_{D}$, exhibits a long tail and is well fit with stretched exponentials: $F(t)=$ $f_{\infty}\left(\tau_{D}\right)-C e^{-\alpha t^{\beta}}$ with $\beta \approx 0.4$ (dotted lines). Even though the fits are based on runs up to $t=300,000$ for $\tau_{D}=1.65$ and $\tau_{D}=1.7$, they do not provide a value of $f_{\infty}\left(\tau_{D}\right)$ accurate enough for establishing whether true persistent activity exists for a small fraction of the network realizations $\left(0.97 \leq f_{\infty}\left(\tau_{D}=1.65\right) \leq 1\right)$. Strikingly, the dependence of $F(t)$ on the delay time exhibits a high degree of structure, suggestive of 'resonances' at values of $\tau_{D}$ for which some chains and trees can be optimally utilized.

One of the salient features of the emergent dynamics of the model is persistent self-sustained activity at low densities $p$ of shortcuts. In this regime, the network is in fact bistable between 'off' and 'on' states, and can be switched between them with sufficiently large stimuli, as illustrated in fig [6] The synchronous stimulation of a sufficiently large number of neurons while the network is in the 'on' state increases the level of activity and effectively pushes the network to the right of the failure transition (cf. fig 2), causing a transition into the 'off' state.

To address the fact that real neuronal systems are noisy, the simulation shown in fig [6 includes Gaussian fluctuations in the membrane potential; their amplitude is chosen so as to cause the neurons to spike irregularly at a low rate. To keep the noisy spiking of a single neuron from generating a traveling pulse and initiating the 'on' state, we adjust the synaptic conductance so that several adjacent neurons must fire in rapid succession in order to 


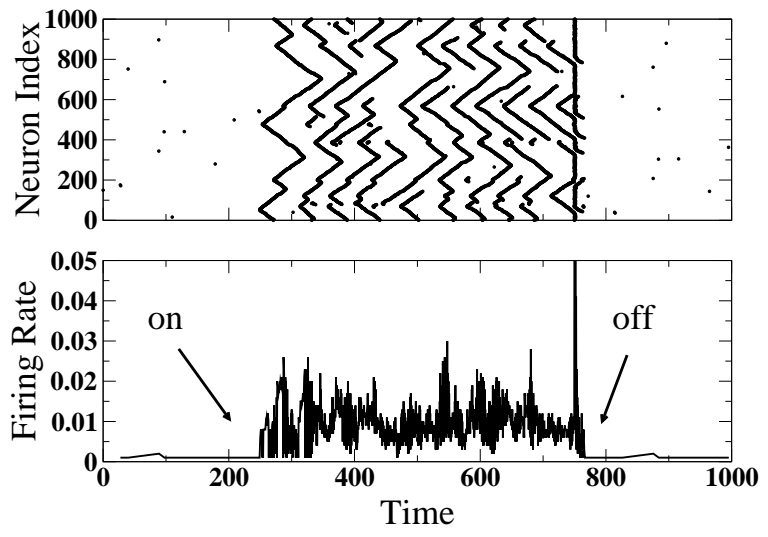

FIG. 6: Raster plot (top) and instantaneous firing rate (bottom) for $p=0.10$ and $k=5$. About 10 adjacent neurons are stimulated synchronously at $t=250$; about $20 \%$ of the neurons are activated at $t=750$.

propagate a pulse of excitation. The network topology is modified accordingly: we extend the local coupling to include up to $2 k$ neighbors $\left(w_{i, i \pm j}=1\right.$ for $\left.j=1 \ldots k\right)$ and model long-range connections via a population of intermediate excitatory neurons which both receive input from and project to multiple adjacent neurons. Under these conditions, spontaneous activity is highly unlikely to initiate traveling pulses. However, a sufficiently large stimulus, synchronous across several neurons, can again turn the state of elevated activity on and off (cf. fig [6); bistability is thus robust with respect to noise.

Network bistability has been hypothesized to be the neural correlate underlying the type of short-term memory known as working memory in the prefrontal cortex of monkeys and humans. Much more realistic and physiologically plausible models of cortical layers have been studied within the context of working memory (e.g. [17]). Yet, not much attention has been given to heterogeneities in network topology or to long-range excitatory connections. The work presented here suggests that closer at- tention be given to the role of connectivity as an additional factor that contributes to the generation of the persistent, active state associated with working memory.

In conclusion, we have investigated the effect of incorporating random unidirectional shortcuts to a onedimensional network of locally coupled integrate-and-fire neurons. We find that even a very low density of shortcuts suffices to generate persistent activity from a local stimulus through the reinjection of activity into previously excited domains. As the density of shortcuts is increased, the substantial decrease in the effective system size characteristic of small-world networks causes a crossover into a regime characterized by failure to sustain activity for essentially all network configurations. For sufficiently slow propagation velocities of the activity and sufficiently high shortcut densities, an intriguing second crossover occurs into a regime in which the activity still fails but only after often exceedingly long and strongly chaotic transients.

The complex dynamical phenomena we find in this extremely simple model are based on a robust mechanism: propagating pulses of activity that are sustained by branching and reinjection. We therefore expect that more realistic models of neuronal networks, which may include multiple ion channels and continuous synaptic currents as well as inhibitory coupling, will show qualitatively the same behavior upon the addition of shortcuts if they originally support propagating pulses of activity [18] that annihilate upon collision. Preliminary computations show that the failure transition persists if shortcuts are not allowed to exceed a maximal length $L_{m}$, as long as $L_{m} \tau_{D}>T_{R}^{(1)}[19]$. The phenomena should also be accessible in excitable chemical systems 20], in which the shortcuts could be implemented using video feedback.

We gratefully acknowledge support from the Department of Energy (DE-FG02-92ER14303), NSF grant DMS-9804673, and the NSF-IGERT program Dynamics of Complex Systems in Science and Engineering (DGE9987577).
[1] D. J. Watts and S. H. Strogatz, Nature 393, 440 (1998).

[2] S. H. Strogatz, Nature 410, 268 (2001).

[3] R. Albert and A. L. Barabasi, Rev. Mod. Phys. 74, 47 (2002).

[4] A. Pekalski, Phys. Rev. E 64, 057104 (2001).

[5] A. D. Sánchez, J. M. López, and M. A. Rodríguez, Phys. Rev. Lett. 88, 048701 (2002).

[6] M. Barahona and L. M. Pecora, Phys. Rev. Lett. 89, 054101 (2002).

[7] R. Pastor-Satorras and A. Vespignani, Phys. Rev. Lett. 86, 3200 (2001).

[8] V. B. Mountcastle, Brain 120, 701 (1997).

[9] G. González-Burgos, G. Barrionuevo, and D. A. Lewis, Cereb. Cortex 10, 82 (2000).

[10] D. A. McCormick and D. Contreras, Annu. Rev. Physiol. 63, 815 (2001).
[11] J. Y. Wu, L. Guan, and Y. Tsau, J. Neurosci. 19, 5005 (1999).

[12] R. Demir, L. B. Haberly, and M. B. Jackson, J. Neurophys. 86, 2445 (2001).

[13] D. S. Melchitzky, G. González-Burgos, G. Barrionuevo, and D. A. Lewis, J. Comp. Neurol. 430, 209 (2001).

[14] L. F. Lago-Fernández, R. Huerta, F. Corbacho, and J. A. Sigüenza, Phys. Rev. Lett. 84, 2758 (2000).

[15] L. F. Lago-Fernández, F. J. Corbacho, and R. Huerta, Neural Networks 14, 687 (2001).

[16] M. E. Newman, C. Moore, and D. J. Watts, Phys. Rev. Lett. 84, 3201 (2000).

[17] A. Compte, N. Brunel, P. S. Goldman-Rakic, and X.-J. Wang, Cereb. Cortex 10, 910 (2000).

[18] D. Golomb and G. B. Ermentrout, Phys. Rev. Lett. 86, 4179 (2001). 
[19] A. Roxin, H. Riecke, and S. A. Solla (unpublished).

[20] K. Martinez, A. L. Lin, R. Kharrazian, X. Sailer, and

H. L. Swinney, Physica D 168, 1 (2002). 